\title{
Chemical Abundances and Milky Way Formation
}

\author{
Gerry Gilmore $^{1}$ and Rosemary F.G. Wyse ${ }^{2}$ \\ 1 Institute of Astronomy, Madingley Road, Cambridge, UK \\ 2 Department of Physics and Astronomy, Johns Hopkins University, Baltimore, MD \\ 21218, USA
}

\begin{abstract}
Stellar chemical element ratios have well-defined systematic trends as a function of abundance, with an excellent correlation of these trends with stellar populations defined kinematically. This is remarkable, and has significant implications for Galactic evolution. The source function, the stellar Initial Mass Function, must be nearly invariant with time, place and metallicity. Each forming star must see a wellmixed mass-averaged IMF yield, implying low star formation rates, with most star formation in at most a few regions of similar evolutionary history. These well-established results are difficult to reconcile with standard hierachical formation models, which assemble many stellar units: galaxy evolution seems to have been dominated by gas assembly, with subsequent star formation. Recent results, and some new ones, on the Galactic bulge, stellar halo and thick disk that justify this conclusion are presented.
\end{abstract}

\section{The Context: abundances and galaxy formation}

The $\Lambda \mathrm{CDM}$ paradigm for structure formation in the Universe, described in many hundreds of published papers, is very effective at reproducing observed large scale structure, based on a boundary condition of a scale-free Gaussian random power spectrum. Yet $\Lambda \mathrm{CDM}$ contains no information on the physics of whatever makes up CDM, and remains deficient in its description of galaxies and small-scale structures: thus it is on galaxy scales and smaller where we can still learn the most, and hopefully attach some (astro-)physics to an ab initio power spectrum.

Among the very many studies which emphasise the current progress in studies of galaxy-scale predictions, we note as recent examples Glazebrook etal (2004) and Cimatti etal (2004), which highlight the buildup of massive spheroids at earlier times than predicted; de Blok (2004) showing CDM mass profiles on small scales are shallower than predicted; D'Onghia \& Lake (2004), on the substructure problem, showing it extends from single galaxies to galaxies in groups; and Abadi etal (2003) illustrating how detailed comparisons of simulations and data for the Solar neighbourhood are becoming feasible.

How will we identify the extra astrophysics required to reconcile the properties of CDM dark haloes with those of luminous galaxies? We can start by developing knowledge of the evolutionary history of at least one place in at least one galaxy. We would be unlucky if that place were far from the norm: alternatively, any theory that predicts such a history to be very unusual might be suspect the galaxian Copernican principle. Kinematics and current spatial location are of course critical parameters, so that traditional stellar populations analyses are 
prerequisities. However, kinematical histories, as represented by velocity dispersions, are at best confused by phase-wrapping over time, and can be largely lost if a virialisation process is associated with significant mergers. Kinematics do provide valuable statistical information, but, apart from the single (approximately, sometimes) conserved quantity of angular momentum, provide direct information only about very recent mergers, a minor aspect of Galaxy formation in any model. The additional complementary information is best available from chemical abundances, especially including element ratios.

It is remarkable that chemical abundances are a valid, and relatively robust, tracer of galactic evolution: it is worth considering why this is possible.

Figure 1 (from Venn etal 2004) is a compilation of most of the recent highquality element ratio data for Galactic field stars (together with data for some stars in several satellite galaxies). The remarkable implication of this figure is that the overwhelming majority of Galactic field stars have element ratios relative to Fe which have a scatter which is always relatively small, over a range of some 4 dex in $[\mathrm{Fe} / \mathrm{H}]$. This small scatter is quite contrary to much simple expectation, and has profound implications.

One implication follows from the continuity in the pattern of the elemental ratios as a function of $[\mathrm{Fe} / \mathrm{H}]$ : at every metallicity, all forming stars have element ratios which require that they are being formed from gas which has a common well-mixed history. At every metallicity, the star-forming gas must be well-mixed, and only mildly different in chemical element-ratio enrichment than that gas which formed the previous stellar generation. This seriously restricts the role of significant inflows of gas over an extended period of time, as inflows of gas with a very different enrichment history would induce scatter. However, inflow of metal-free gas (as often invoked to 'solve' the local disk 'G-dwarf problem') would reduce $[\mathrm{Fe} / \mathrm{H}]$ while leaving $[\alpha / \mathrm{Fe}]$ unchanged. A second implication follows from the dependence of element production on the initial mass of the main-sequence progenitor of the supernova. The observed small dispersion in element ratios, even under the extreme assumption of perfect ISM mixing, requires that each new-forming star sees an approximately invariant, and mass-averaged, IMF (eg Wyse \& Gilmore 1992; Nissen et al. 1994). The rate of star formation must be low enough to allow time for element creation followed by large-scale mixing.

That is, the straightforward interpretation of abundance data for Galactic field stars in terms of stellar populations is feasible only because the Galaxy apparently acquired its gas early, or at a rate which was well-matched to the star formation rate across the whole volume now sampled by local halo stars, and kept this gas well-mixed; and because the stellar IMF is (close to) invariant over time and metallicity. Neither deduction was obvious, nor is the underlying physics understood. However, these two deductions apply so well they have become assumed: authors use any violation to rule out some possible Galaxy merger histories, as in the Venn etal analysis from which Figure 1 is taken.

We now briefly consider in turn the abundance constraints on the major Galactic stellar populations, highlighting the poorly known aspects. 


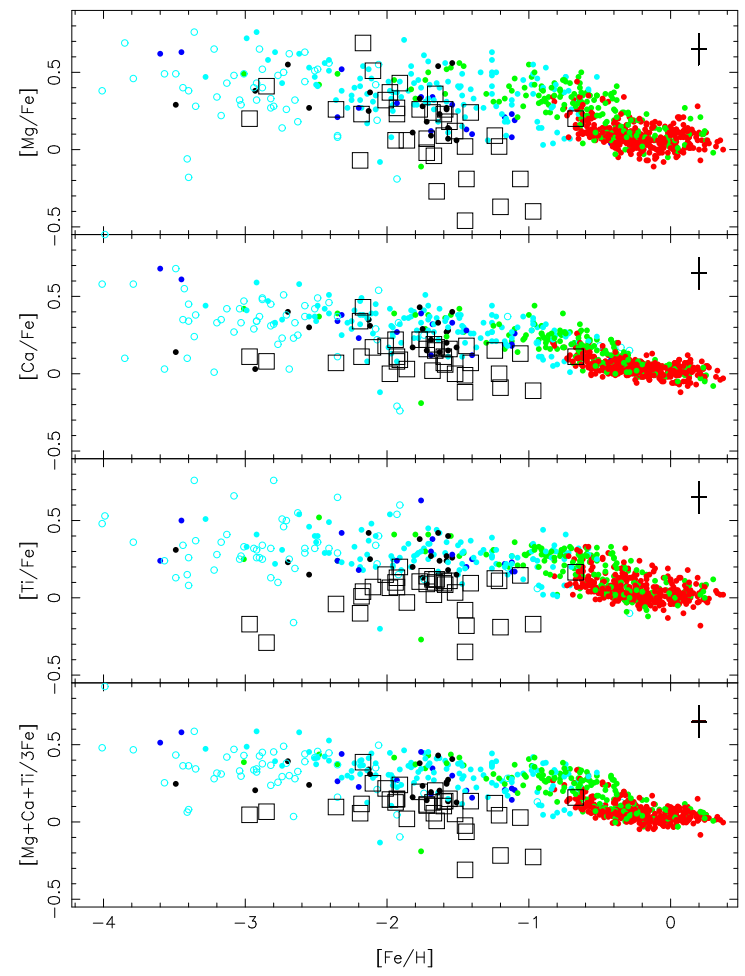

Fig. 1. Element ratio data for Galactic field stars (dots) compiled by Venn etal (2004). [The open squares are stars in dSph galaxies.] The important point to notice here is the very small scatter in element abundances for Galactic field stars at any given $[\mathrm{Fe} / \mathrm{H}]$ value, over 4 dex in $[\mathrm{Fe} / \mathrm{H}]$.

\section{The Galactic Bulge}

It is quite astonishing how little observational information is available on the chemistry and kinematics of the Galactic Bulge. A massive and exciting opportunity for the current multi-object spectrographs is being overlooked here.

The central regions of the Galaxy, including the Bulge, should accumulate any tightly-bound high phase-space density material associated with mergers (since the most-bound regions of substructure end up in the most-bound regions; Zurek, Quinn \& Salmon 1988); low-angular momentum material in general; material driven in by bar asymmetries in the gravitational potential; and any accreted gas which can cool efficiently and lose angular momentum. The earliest stars formed will be there, even in the merger scenario for build-up of bulges (e.g. Kauffman 1996), while the whole will be affected in some as yet undefined way by feedback from the central Super-Massive Black Hole. It should be a very complex place, well worth detailed investigation. The luminosity scale height of the Bulge is of order 300pc, so that even the best studied 'inner' field, Baade's 
Window at coordinates $(\ell=1, b=-4)$ or a projected Galactocentric distance of $\gtrsim 500 \mathrm{pc}$, is itself rather far from the heart of the matter.

In so far as these properties are determined, the outer bulge, beyond 2 scale lengths, is apparently predominately mildly metal-rich (perhaps 0.5 Solar in the mean) with a very broad abundance distribution function, is older than $\sim 10 \mathrm{Gyr}$, ie is indistinguishable from the old metal-rich globular clusters, which may be related, and is alpha-element enhanced (e.g. Ibata \& Gilmore 1995a, 1995b; Sadler, Rich \& Terndrup 1996; Zoccali et al. 2003; Fulbright, Rich and McWilliam 2004): this does not favour merger origin models, but does suggest predominant in-situ star formation. A star formation rate of $10 M_{\odot} y r^{-1}$ is implied. The angular momentum distribution function is dominated by very low angular momentum, and is strongly dissimilar to the corresponding distribution for the Galactic disk: this argues against a formation process through disk-bar evaporation (Figure 2, and cf. Ibata \& Gilmore 1995b), and suggests a close connection between bulge and halo in formation.

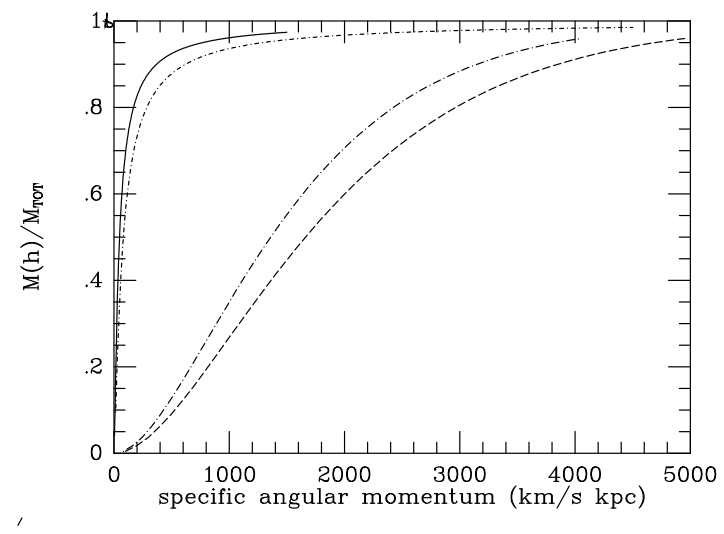

Fig. 2. The distribution of specific angular momentum in the dominant Galactic stellar populations. This indicates the similarity in this fundamental parameter, and presumably in origin, between bulge and halo (the two curves near the top left of the figure), and the quite distinct bahaviour of the Galactic thin and thick disks (the two curves through the centre of the figure). This figure is from Wyse \& Gilmore 1992.

The structure of the inner Bulge, where most of the mass is, is inevitably confused by the inner disk, and by the extremely dense stellar super-cluster with small scale length concentrated at the very centre with its associated black hole (Schodel, Ott, Genzel etal 2003). Recent studies in the IR have hinted at the considerable complexity of the central stellar populations (van Loon, Gilmore, Omont etal 2003), with young high-mass star and cluster formation at solar metallicity (Najarro etal 2004), a vast reservoir of molecular gas to feed continuing star formation (Pierce-Price etal 2000), and complex bar and disk structure (Bissantz, Debattista \& Gerhard 2004). Extant analyses have usually assumed 
the bar is associated with the bulge, rather than with the inner old disk, or, more commonly, do not make any distinction between these various central populations, even though they may well have very different kinematic, age and abundance distribution functions, and have formed in very different ways. Unravelling their inter-relationships is not yet possible due to the limited data. At present one needs to be careful to define terms, such as 'main bulge' (cf. Wyse, Gilmore \& Franx 1997)

Considerable progress is currently being made in mapping the very inner Galaxy. In a recent study, Babusiaux \& Gilmore (2004) have used near-IR photometry to map the inner galaxy in the Galactic Plane (figure 3), using red clump stars as tracers. Their photometric work suggests that the bulge stars 1-deg from the Plane (ie, 4 times closer in than Baade's Window) are metal-rich and alphaenhanced, while the stars which define the bar itself, measured within $0.2 \mathrm{deg}$ of the Plane, best match intermediate-age isochrones without alpha-enhancement, The stars in the Bar itself seem more disk-like than those stars seen in Baade's window and other fields at similar high latitudes. Direct spectroscopic confirmation, to ensure age-metallicity degeneracies are not confusing the photometric analyses, should be possible, now that the individual bar clump giants have been identified.

\section{The Stellar Halo}

Figure 2 above illustrates a similarity in at least one fundamental parameter between the Bulge and the stellar halo. The stellar halo traced by stars passing near the Sun is some $30 \%$ of its total mass of $\sim 2 \times 10^{9} M_{\odot}$. These stars are predominantly old and metal poor. They show remarkable uniformity in their relationship between the element ratios and the total metal abundance (Figure 1), with implications noted above.

It has been known for many years (eg Unavane et al 1996; Gilmore \& Wyse 1998), and has been re-emphasised recently (this meeting) based on much better data, that the stars in the stellar halo are unlike those in surviving Galactic satellites. Considerable progress is being made in determining the properties of the current Galactic satellites, especially the fundamental properties, mass density and dark matter profile, as well as in analysing the tracers of star formation histories, CMDs and chemical abundances. All the dSph satellites will have good kinematic maps complete within the near future, almost all based on FLAMES data, and all providing useful chemical abundance maps (UMi: Kleyna etal 2003; Draco: Kleyna etal 2001; Sextans: Kleyna etal 2004; LeoI, LeoII, Carina: Harbeck etal this meeting; Gilmore etal in prepn; Sgr: Ibata, Wyse, Gilmore etal 1997; Scl: Tolstoy etal, this meeting; Fornax: Tolstoy etal in prepn). All have similarities and complexities, yet none provides a source for a 'typical' field halo star. The different patterns of elemental abundances in the dwarf galaxies, which apparently rules them out as 'building blocks' for the Milky Way, can be understood in terms of their different star formation histories (cf. Gilmore \& Wyse 1991). 
This suggests strongly that the stellar halo formed in place early in Galactic history - a conclusion apparently required by the data, yet strongly in disagreement with most current galaxy formation simulations. The simulations imply significant late accretion of galaxies into the Milky Way: do we see this? A discovery which encouraged much current enthusiasm was that of the Sgr dwarf (Ibata, Gilmore \& Irwin 1994, 1995): it is salutary to recall that Sgr was recognised as peculiar in real time at the AAT (during the Ibata/Gilmore bulge study) because both its velocity and its stellar populations were quite unlike any expected (or observed) field star. Sgr does however single-handedly make a substantial perturbation to the stellar mass (and age mix) of the stellar halo. More recently a second, and possibly a third, structure has been identified in the outer halo from SDSS photometry (Newberg et al. 2002); their relationship to the Sgr dwarf is unclear, but most confirmed structure may be ascribed to this galaxy (cf. Majewski et al. 2003). A firm conclusion is that in another Hubble time, the stellar halo will be relatively younger than it is today (Wyse 1996).

The halo globular cluster system also provides valuable information, since accurate distances, and hence reliable ages, can be derived. Mackey \& Gilmore (2004) recently acquired and compiled a new, nearly complete, internally consistent set of photometric studies of the globular cluster population in both the Milky Way and its satellite galaxies: they deduce, from analysis of HB morphology, age, abundance and structural information, somewhat more relaxed limits on accretion of the halo than earlier analyses, but still preclude late accretion as a dominant factor.

Study of the relative importances of assembly of stellar systems and in situ star formation remains extremely active, and will presumably soon converge as the large area CCD photometric surveys cover the whole sky, and accurate spectroscopic surveys begin to acquire large samples.

\subsection{M31 and its halo/bulge/thick disk}

An interesting case here is M31, which has long been suspected of having tidally stripped M32 (Faber 1973; Choi, Guhathakurta \& Johnson 2002), and now we have kinematic evidence that it is also currently accreting from NGC 205 (McConnachie et al. 2004). However, the equivalent of the Milky Way's halo population II, a metal-poor component to the 'halo', traced by RR Lyrae stars and by metal-poor giants, is not dominant even at very many photometric scale lengths from the centre of M31 (e.g. Durrell, Harris \& Pritchet 2004). The relation to accreted sateliites is clearly complex (e.g. Ferguson et al. 2002). The dominant non-thin-disk population in M31 is rather metal-rich, with a mean of $\sim-0.6$ dex (Mould \& Kristian 1986; Durrell et al. and references therein). Wyse \& Gilmore (1988) discuss the apparent equivalence between this extended intermediate population in M31 and the Galactic thick disk, an equivalence which has become stronger as studies of M31 have developed (Sarajedini \& van Duyne 2001; Brown et al. 2003). A fuller interpretation in terms of the mass assembly history of M31 awaits the current wide-area coverage with a combination of deep CMDs for age constraints, together with spectroscopic kinematics and metallicities. 


\section{The Old Disks}

The thick disk probably was caused by, and/or is the remnant of, one or more (early?) merger events. The (local) mean thick disk metallicity of - 0.6 dex and its old age suggest a very massive satellite - comparable at least to the LMC? - was destroyed if the (local) thick disk is its remnant. Simulations (eg Abadi etal 2003) suggest at least the old thick disk will be the remnant of several merged subgalaxies, with the relative mix changing with radius. This may be consistent with recent AAT 2dF survey results (Gilmore, Wyse \& Norris 2002) which suggest that the distribution function of angular momentum, and perhaps metallicity, a few kpc from the Plane is not consistent with simple local extrapolations.

One of the more challenging things to understand in these multiple-fragment models is the evolution of the present-day surviving thin old disk, particularly since a recent accretion of the thick disk could destroy the thin disk. Further, in these models stars should not form in a thin disk at all until after most of the merging is complete, since otherwise disks have too small a scale-length (Navarro \& Steinmetz 1997). A solution to both these issues may be that the local old thin disk is also accreted in these models. Here the recent results of many authors (cf Figure 1 above) have shown there is a very small scatter in element ratios at any $[\mathrm{Fe} / \mathrm{H}]$ value, particularly within a stellar population, yet there is an extremely large scatter in the age-[Fe/H] relation at every age (Nordstrom et al. 2004). Linking the local and global remains a challenge.

\section{References}

1. Abadi, M., Navarro, J., Steinmetz, M. \& Eke, V. 2003 ApJ 59721

2. Babusiaux, C., \& Gilmore, G. 2004 MNRAS submitted

3. Bissantz, N., Debattista, V. \& Gerhard, O. 2004 ApJ 601 L155

4. Brown, T. et al. 2003 ApJL 592 L17

5. Choi, P., Guhathakurta, P. \& Johnson, K. 2002 AJ 124, 310

6. Cimatti. A., Daddi, E., Renzini, A., Vanzella, E., et al. 2004 Nature 430184

7. de Blok, W.J.K. 2004, IAU Symp 220 eds Ryder et al. (ASP, San Francisco) p69

8. D’Onghia, E., \& Lake, G., 2004 ApJ 612628

9. Durrell, P., Harris, W. \& Pritchet, C. 2004 AJ 128260

10. Faber, S.M. 1973 ApJ 179423

11. Ferguson, A. et al. 2002 AJ 1241452

12. Fulbright, J., Rich, R.M. \& McWilliam, A., 2004 astroph-0411041

13. Gilmore, G., Wyse, R.F.G. 1998 AJ 116748

14. Gilmore, G., Wyse, R.F.G. \& Norris, J.E. 2002 ApJL 574 L39

15. Glazebrook, K., Abraham, R., McCarthy, P. et al. 2004 Nature 430181

16. Ibata, R., Gilmore, G. \& Irwin, M. 1994 Nature 370194

17. Ibata, R. \& Gilmore, G. 1995a MNRAS 275, 591

18. Ibata, R. \& Gilmore, G. 1995b MNRAS 275, 605

19. Ibata, R., Gilmore, G. \& Irwin, M. 1995 MNRAS 277781

20. Ibata, R., Wyse, R.F.G., Gilmore, G., Suntzeff, N. \& Irwin, M. 1997 AJ 113634

21. Ibata, R. et al. 2004 MNRAS 351117

22. Kleyna, J., Wilkinson, M., Evans, N.W. \& Gilmore, G. 2001 ApJ 563 L115 
23. Kleyna, J., Wilkinson, M., Evans, N.W. \& Gilmore, G. 2004 astroph-0409066

24. Kleyna, J., Wilkinson, M., Gilmore, G. \& Evans, N.W. 2003 ApJ 588 L21

25. Kauffmann, G. 1996 MNRAS 281487

26. van Loon, J., Gilmore, G., Omont, A. et al. 2003 MNRAS 338857

27. Mackey, A.D. \& Gilmore, G. 2004 MNRAS 354470

28. Majewski, S., Strutskie, M., Weinberg, M. \& Ostheimer, J. 2003 ApJ 5991082

29. McConnachie, A. et al. 2004 MNRAS 351 L94

30. Mould, J. \& Kristian, J. 1986 ApJ 305591

31. Najarro, F., Figer, D., Hillier, D. \& Kudritzki, R. 2004 ApJ 611 L105

32. Navarro, J. \& Steinmetz, M. 1997 ApJ 47813

33. Nissen, P. E., Gustafsson, B., Edvardsson, B., Gilmore, G. 1994 A\&A 285440

34. Newberg, H., Yanny, B., Rockosi, C., etal 2002 ApJ 569245

35. Nordstrom, B., Mayor, M., Andersen, J. etal. 2004 A\&A 418989

36. Pierce-Price, D., et al. 2000 ApJ 545 L121

37. Reitzel, D., Guhathakurta, P. \& Rich, R.M. 2004 AJ 1272133

38. Sadler, E., Rich, R.M. \& Terndrup, D. 1996, AJ 112171

39. Sarajedini, A. \& van Duyne, J. 2001, AJ 1222444

40. Schodel, R., Ott, T., Genzel, R. et al. 2003 ApJ 5961015

41. Unavane, M., Wyse, R.F.G. \& Gilmore, G. 1996 MNRAS 278727

42. Wyse, R.F.G. 1996 ASP Conf series 88, eds Trimble \& Reisenegger p128

43. Wyse, R.F.G. \& Gilmore, G. 1988 AJ 951404

44. Wyse, R.F.G. \& Gilmore, G. 1992 AJ 104144

45. Wyse, R.F.G., Gilmore, G. \& Franx, M. 1997 ARAA 35637

46. Zoccali, M. et al. 2003 A\&A 399931

47. Zurek, W., Quinn, P. \& Salmon, J. 1988 ApJ 330519 


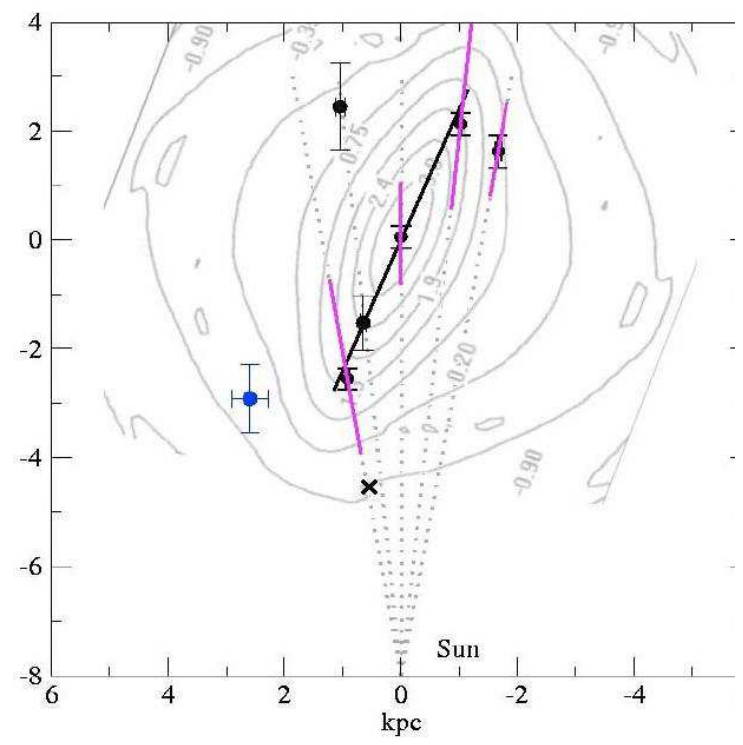

Fig. 3. The 2-D structure of the inner Galactic bar, based on direct photometric analysis of red clump stars as distance indicators. Now these individual bar stars are identified, detailed study of their kinematics and chemical abundances will be possible. Preliminary photometric indications suggest the bar is more closely disk than bulge in its populations. This figure is from Babusiaux \& Gilmore 2004. 